\title{
Pressure overload leads to coronary plaque formation, progression, and myocardial events in $\mathrm{ApoE}^{-/-}$mice
}

\author{
Alice Marino, ${ }^{1,2,3}$ Yi Zhang, ${ }^{1,2,3}$ Luisa Rubinelli, ${ }^{1,2,3}$ Maria Antonietta Riemma, ${ }^{1,2,3,4}$ James E. Ip, ${ }^{5}$ \\ and Annarita Di Lorenzo 1,2,3 \\ 'Department of Pathology and Laboratory Medicine, ${ }^{2}$ Cardiovascular Research Institute, and ${ }^{3}$ Brain and Mind Research \\ Institute, Weill Cornell Medicine, New York, New York, USA. ${ }^{4}$ Department of Pharmacy, School of Medicine, University \\ of Naples "Federico II," Naples, Italy. 'Division of Cardiology, Department of Medicine, Weill Cornell Medicine, New York \\ Presbyterian Hospital, New York, New York, USA.
}

\begin{abstract}
Hypercholesterolemia and hypertension are two major risk factors for coronary artery diseases, which remain the major cause of mortality in the industrialized world. Current animal models of atherosclerosis do not recapitulate coronary plaque disruption, thrombosis, and myocardial infarction occurring in humans. Recently, we demonstrated that exposure of the heart to high pressure, by transverse aortic constriction (TAC), induced coronary lesions in $\mathrm{ApoE}^{-/-}$mice on chow diet. The aim of this study was to characterize the magnitude and location of coronary lesions in $\mathrm{ApoE}^{-/-}$mice after TAC and to assess the susceptibility of coronary plaque to disruption, leading to myocardial events. Here, we describe a reliable pathological condition in mice characterized by the development of coronary lesions and its progression, leading to myocardial infarction; this model better recapitulates human disease. Following TAC surgery, about $90 \%$ of $\mathrm{ApoE}^{-/-}$mice developed coronary lesions, especially in the left anterior descending artery, with $59 \%$ of the mice manifesting a different magnitude of LAD stenosis. Myocardial events, identified in $74 \%$ of the mice, were mainly due to coronary plaque thrombosis and occlusion. That TAC-induced development and progression of coronary lesions in $\mathrm{ApoE}^{-/-}$mice, leading to myocardial events, represents a potentially novel and important tool to investigate the development of coronary lesions and its sequelae in a setting that better resemble human conditions.
\end{abstract}

Conflict of interest: The authors have declared that no conflict of interest exists.

Copyright: (c) 2019 American Society for Clinical Investigation

Submitted: February 18, 2019 Accepted: March 27, 2019 Published: May 2, 2019.

Reference information: /CI Insight. 2019;4(9):e128220. https://doi. org/10.1172/jii.insight.128220.

\section{Introduction}

Coronary artery disease (CAD) remains the leading cause of morbidity and mortality in the US, accounting for more than $40 \%$ of deaths due to cardiovascular disease. Statins have been partially successful, suggesting the need for alternative therapeutic options (1). In this regard, the development of novel therapeutic approaches is impeded, in part, by the use of animal models of atherosclerosis that do not fully mirror the features of human CAD (2).

Since 1994, $\mathrm{ApoE}^{-/-}$mice (3) have been widely used to study the pathogenesis of atherosclerosis and to develop pharmacologic and other interventional therapeutic approaches (4-6). In spite of high plasma cholesterol (6), ApoE ${ }^{-/-}$mice develop minimal atherosclerosis on a regular diet (3). Thus, to induce atherosclerosis, ApoE ${ }^{-/-}$mice have been fed with Western-type diet (3), resulting in plasma cholesterol levels $\geq 1400 \mathrm{mg} / \mathrm{dl}$, a level which is markedly higher than those reported in humans (7). Subsequently, the same approach was used for LDL receptor-knockout $\left(\mathrm{Ldlr}^{--}\right)$mice (8). ApoE ${ }^{-/-}$and $\mathrm{Ldlr}^{--}$mice fed high-fat diets develop extensive lesions in the aortic root, the aorta, and its principal branches; however, they fail to develop atherosclerosis in the coronary arteries, a major affected site in humans (9). Moreover, in these models coronary plaque progression and its sequelae are not observed.

To overcome this drawback and induce coronary lesions, genetic mouse models were generated by excising the srb1 gene (scavenger receptor class B type I, also known as SR-BI) (10) and the Ldlr gene (11) on an $\mathrm{ApoE}^{-/-}$background. However, long-term high-fat diet was still required to induce coronary atherosclerosis in the $\mathrm{Ldlr}^{-/} / \mathrm{ApoE}^{-/-}$mice and remained not sufficient to elicit myocardial infarction (MI). Indeed, additional hypoxic or mental stress was necessary to cause MI, which occurred in only $40 \%$ of 
the mice (11). $\mathrm{SRBI}^{-/-}-\mathrm{ApoE}^{-/-}$mice developed coronary lesions on regular diet, but they had an incredible short lifetime ( 8 weeks) due to a complex pathological condition, making this model not amenable to preclinical studies (10). Other attempts have been made to induce coronary lesions in mice, such as the heterozygous mutation of fibrillin-1 gene on an $\mathrm{ApoE}^{-/-}$background (12). Although coronary lesions formed, stroke was the primary cause of death in more than $60 \%$ of the mice (12). The loss of endothelial nitric oxide synthase (eNOS) was found to enhance atherosclerosis in Western-type diet-fed ApoE ${ }^{-/-}$mice and induce a complex cardiovascular disease, with the formation of distal coronary atherosclerosis, spontaneous aortic aneurysms, and dissections (13). The systemic loss of the serine-threonine protein kinase AKT1 also enhanced atherosclerosis in Western diet-fed $\mathrm{ApoE}^{-/-}$mice and was associated to the presence of coronary lesions (14). Thus, clinically relevant events of coronary plaque rupture and thrombosis cannot be systematically studied in existing mouse models, presenting a complex cardiovascular disease and the lack of reliable coronary lesion formation and progression.

Established risk factors for CAD are hypercholesterolemia and high blood pressure $(15,16)$. In line with clinical findings, transverse aortic constriction (TAC) in $\mathrm{ApoE}^{-/-}$mice, on regular chow diet, induced the development of atherosclerotic lesions in the vascular segments exposed to high pressure, including right carotid artery and coronary arteries, but not in the vessels at low pressure (i.e., left carotid artery and descending aorta) (17). The aim of this study was to define the magnitude and location of coronary lesions in $\mathrm{ApoE}^{-/-}$mice after TAC and to investigate whether the susceptibility of coronary plaque to disruption was reproducible and accompanied by "human-like" events, such as MI and/or sudden death.

Here, we report that TAC-induced coronary atherosclerosis can progress to plaque thrombosis, occlusion, and MI. At 8 weeks after TAC, more than $90 \%$ of $\mathrm{ApoE}^{-/-}$mice developed atherosclerotic lesions in the coronary arteries, particularly in the left anterior descending artery (LAD). About $60 \%$ of the mice presented LAD stenosis.

When subjected to physical stress conditions (i.e., treadmill), more than $70 \%$ of the TAC-operated $\mathrm{ApoE}^{-/-}$mice died and the majority showed striking evidence of previous MI. Systematic and robust histological analysis revealed that MI was caused mainly by coronary occlusion or plaque rupture and/or erosion.

Our study demonstrates, for the first time to our knowledge, that $\mathrm{ApoE}^{-/-}$mice are susceptible to coronary lesion formation and its sequela if chronically exposed to elevated cholesterol $(\sim 350 \mathrm{mg} / \mathrm{ml})$ and increased pressure afterload. The formation of coronary lesions in $\mathrm{ApoE}^{-/-}$mice following pressure overload makes them of great experimental value. The combined phenotype (high cholesterol/pressure overload with coronary lesions) provides a baseline against which either detrimental or protective genetic and environmental factors, as well as drugs targeting atherosclerosis, can be investigated.

\section{Results}

Characterization and distribution of coronary lesions in $A p o E^{-/-}$mice at 8 weeks after TAC. TAC surgery was performed on $\mathrm{ApoE}^{-/-}$mice (about $25 \mathrm{~g}$ of body weight), leading to transthoracic pressure gradient of circa $60 \mathrm{mmHg}$ (Supplemental Figure 1; supplemental material available online with this article; https://doi. org/10.1172/jci.insight.128220DS1). Different parameters were evaluated at different time points, including magnitude and location of coronary lesions, ECGs, echocardiography, and histology of the hearts (Figure 1A). At 8 weeks after TAC, histological analysis of heart sections from the aortic valve (AV) to the apex was performed to identify the presence of coronary lesions, the degree of stenosis, and the lipid content of the plaques (Figure 1, B-E). The majority of early LAD lesions and its branches $(\sim 80 \%)$ presented lipid deposition in both intima and media (Figure 1B and Supplemental Figure 1A). Macrophages with different degrees of lipid accumulation were observed in the intima and in the media (Figure 1B, early lesions, arrows and arrowheads, respectively). In $20 \%$ of the early lesions, the accumulation of the lipids was limited to the intima or to the media (Supplemental Figure 2A). Just as endothelial cells (ECs), activated smooth muscle cells (SMCs) can express adhesion molecules, such as intercellular adhesion molecule (ICAM) and vascular cell adhesion molecule (VCAM), and therefore can interact and retain monocytes/macrophages into the atherosclerotic artery (18). Interestingly, the initial accumulation of lipids into the coronary wall seems to be different throughout the coronary tree. Specifically, the media of smaller coronary arteries in the apex of the heart (i.e., distal LAD and marginal and diagonal arteries) presented greater lipid accrual compared with the proximal LAD (Supplemental Figure 2B), suggesting a different role of SMCs in distinct coronary artery segments during the initial phase of atherosclerosis. Altogether, these findings suggest similar aspects in the onset of coronary atherosclerosis in mice and humans, including the initial local 
A
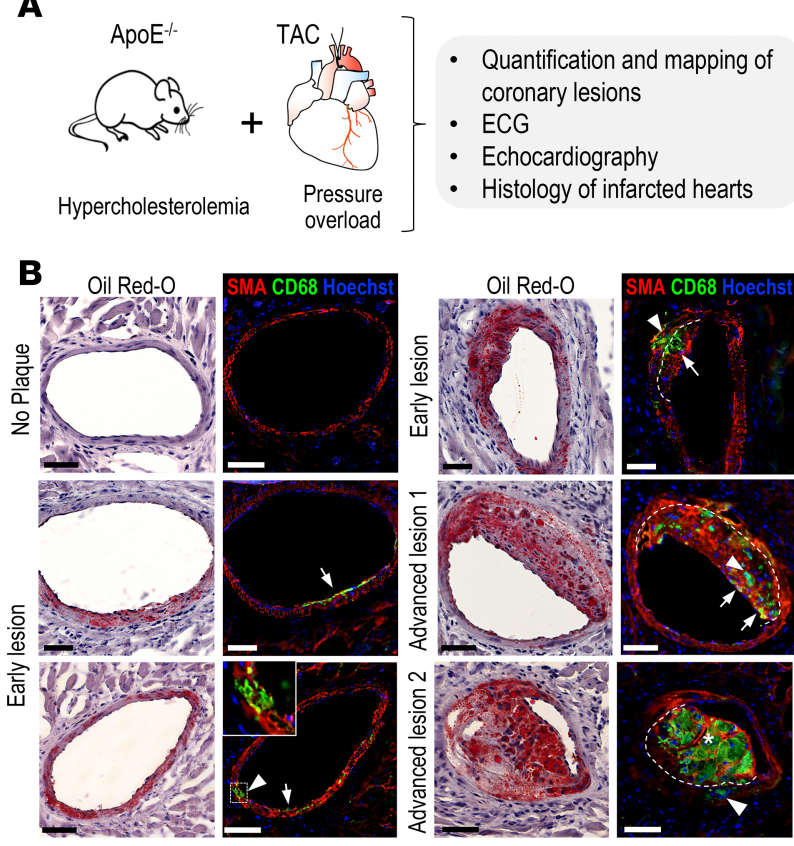

H

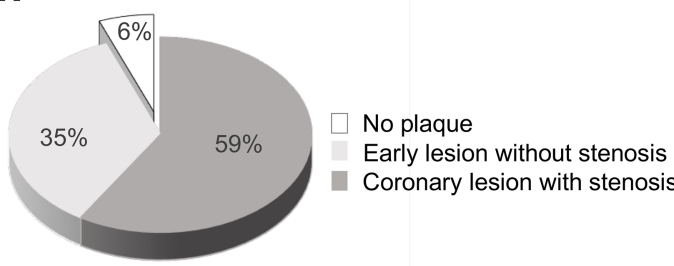

TAC-operated $\mathrm{ApoE}^{-/-}$mice $=34$
C

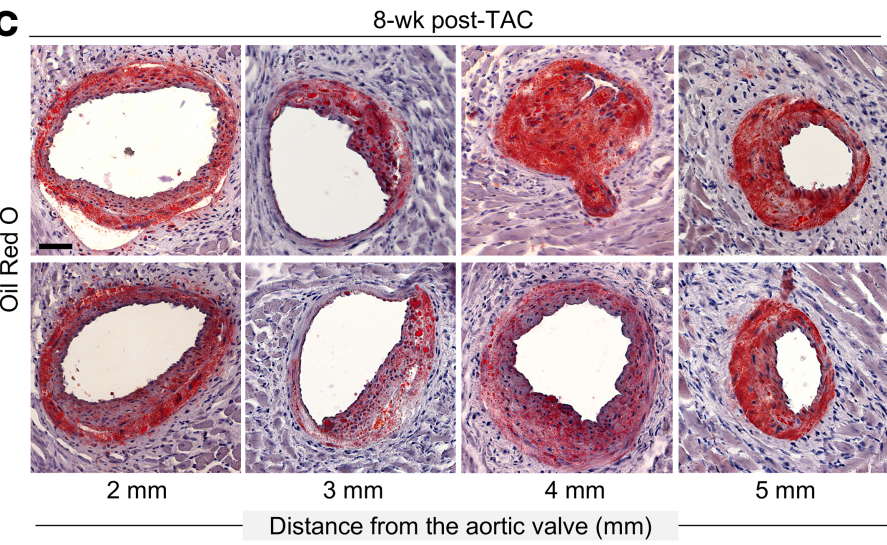

D

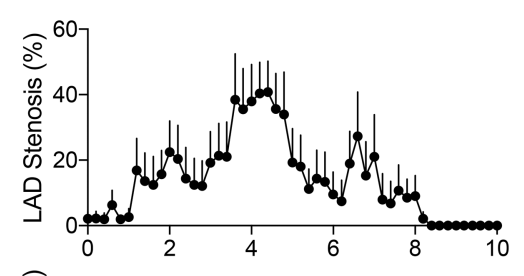

E

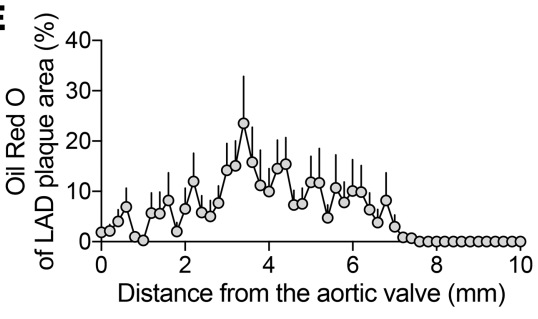

$\mathbf{F}$

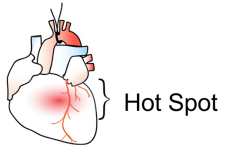

Coronary stenosis

G

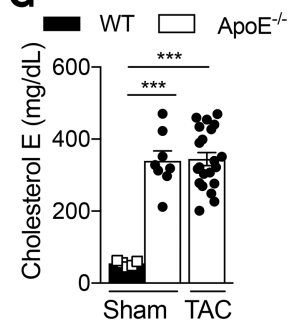

Figure 1. Characterization and distribution of coronary lesions in $\mathbf{A p o E}^{-/-}$mice after TAC. (A) Scheme of the experimental design. Transverse aortic constriction (TAC) was performed in male ApoE $\mathrm{E}^{-/-}$mice ( $25 \mathrm{~g}$ of body weight) on chow diet, followed by histological, echocardiographic, and electrophysiological analysis at different time points after TAC. (B) At 8 weeks after TAC, sequential myocardial sections were stained with Oil Red 0 (binding to lipids) and hematoxylin and immunofluorescently stained with aSMA (SMCs), CD68 (monocyte/macrophages), and Hoechst (nuclei). Oil Red $\mathrm{O}$ showed lipid accumulation in the vascular wall, including endothelial cells (ECs), SMCs, and macrophages, of early and advanced coronary lesions. Consecutive heart sections immunofluorescently labeled showed macrophage (green) infiltration into the intima (white arrows) and media (white arrowheads) of the coronary arteries Scale bar: $50 \mu \mathrm{m}$. (C) Representative images of the coronary plaques in the LAD of 8-week TAC-operated $\mathrm{ApoE}^{-/-}$mice at different distance from the aortic valve (AV) showed different degree of stenosis. Scale bar: $50 \mu \mathrm{m}$. (D) Quantification of LAD stenosis from the AV to the apex of 8-week TAC-operated ApoE ${ }^{-1-}$ mice $(n=10)$. (E) Base-to-apex lipid accumulation in the same LAD lesions, expressed as a percentage of the plaque area $(n=10)$. (F) Cartoon of the hot spot occurrence in coronary plaques. (C) Total plasma cholesterol levels of WT $(n=5)$, sham-operated, and TAC ApoE $E^{-/-}$mice $(n=8$ and 20, respectively; plasma were collected between 1 and 11 weeks after surgery). (H) The pie chart shows the percentage of $\mathrm{ApoE}^{-1-}$ mice with no lesions, early lesions with no stenosis, and coronary lesions with stenosis $(n=34)$. ***P $<0.001$, 1-way ANOVA.

accumulation of lipids in the intima and media, neointimal thickening, and recruitment of macrophages, which later develop into foam cells.

The majority of advanced coronary lesions presented a marked neointimal thickening with lipid accumulation and different degree of macrophage infiltration. Figure 1B shows 2 examples of advanced lesions: one causing $<50 \%$ of luminal stenosis, with pronounced neointimal thickening and infiltrated macrophages (advanced lesion 1), and another presenting $>50 \%$ of stenosis (advanced lesion 2), with robust macrophage infiltration forming foam cells in the intima (asterisks) as well as few in the media (arrowhead). At the same time point, the overall blood cell counts of sham-operated $\mathrm{ApoE}^{-/-}$and TAC-operated ApoE ${ }^{-/-}$and WT mice were not different (Supplemental Figure 3).

Next, 8 weeks after TAC, we quantified the extent of LAD stenosis in sequential myocardial sections from the $\mathrm{AV}$ to the apex (Figure 1, C and D). The highest stenosis was observed along the proximal 
A

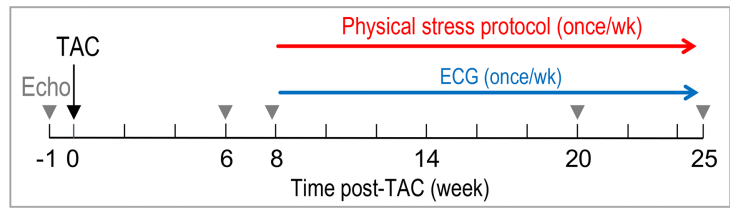

B

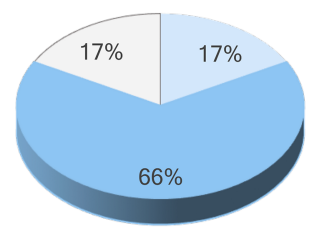

TAC-operated $\mathrm{ApoE}^{-/-}$mice $=35$

D

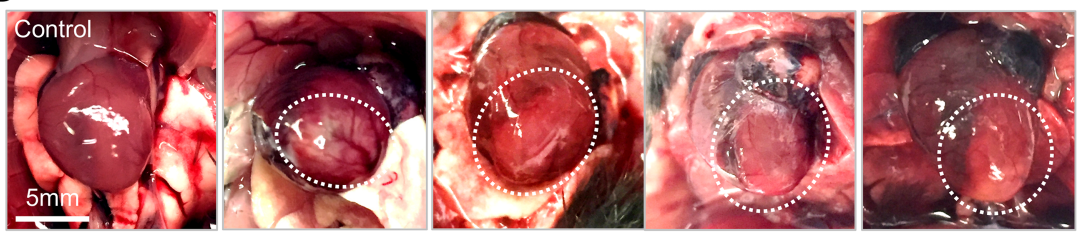

$\left.\begin{array}{l|l}\square & \text { Survived } \\ \text { Dead before } \\ \text { physical stress protocol }\end{array}\right] 34 \%$

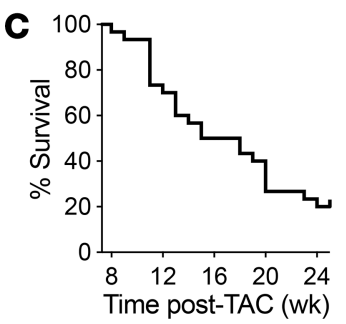

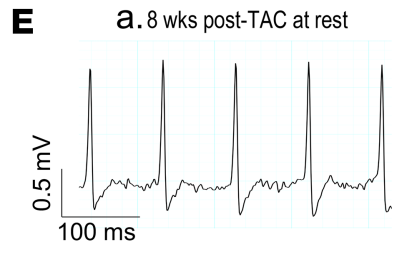

F

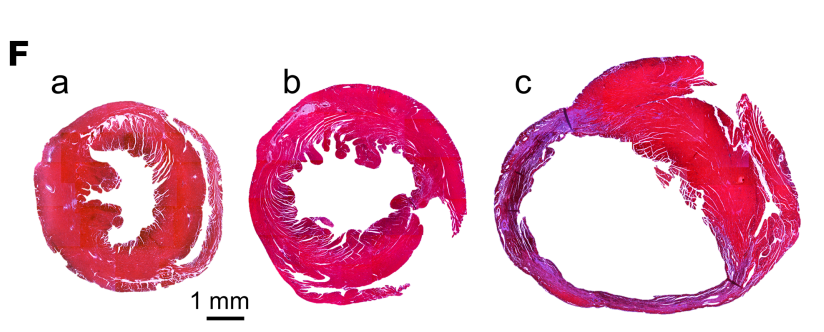

b. 9 wks post-TAC at rest

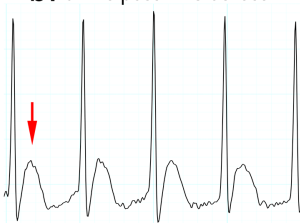

C. 9 wks post-TAC after $3 \mathrm{~min}$ of run

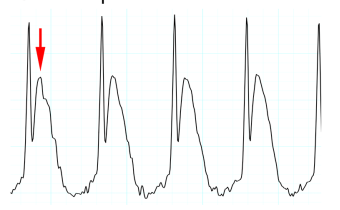

d. 9 wks post-TAC after 4 min $20 \mathrm{sec}$ of run ST elevation and bradycardia
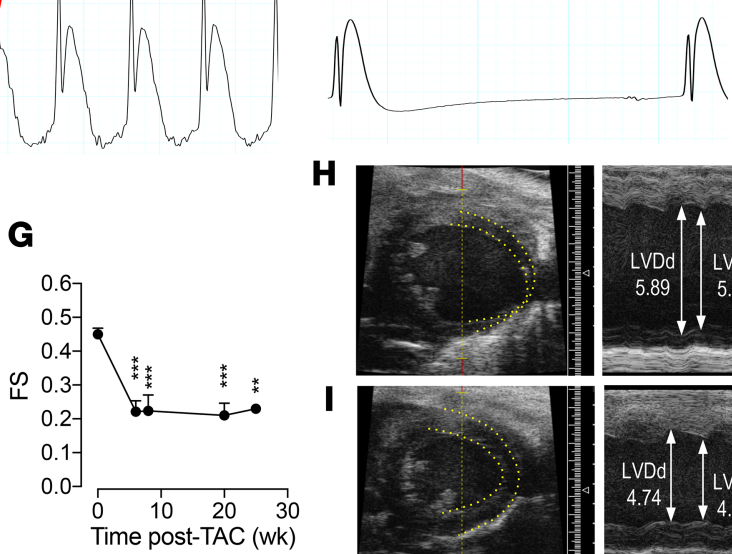

H
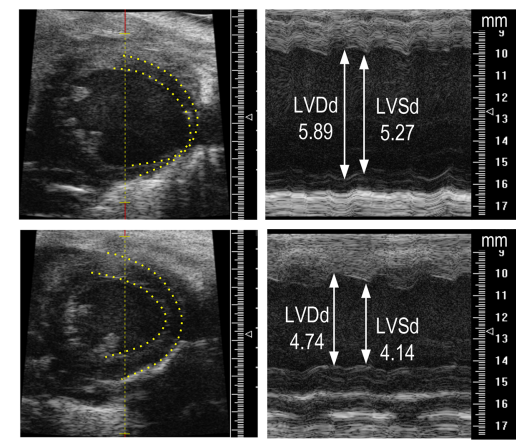

Figure 2. Physical stress induces myocardial events in ApoE-/- mice after TAC. (A) Scheme of the physical stress-induced MI protocol: starting at 8 weeks after TAC, once per week, ApoE ${ }^{-/-}$mice were physically stressed by a treadmill run (4 minutes, at $13 \mathrm{~m} / \mathrm{min}$ ). ECG traces were recorded at baseline and during and 48 hours after the run on the treadmill by using radiotelemetry devices. Echocardiographic analysis was performed at baseline and at 6, 8, 20, and 25 weeks after TAC, as indicated by the gray arrowheads. (B) Percentage of mice that died before and during the stress test protocol and that survived between 8 and 25 weeks after TAC $(n=35)$. (C) Survival curve of ApoE ${ }^{-1-}$ mice after TAC dead during the physical stress-induced MI protocol (from 8-25 weeks after TAC; $n=29$ ). (D) Images of the infarcted ApoE $E^{-/-}$hearts at postmortem examination. Dotted circles indicate the pale myocardium, indicative of infarct. Scale bar: $5 \mathrm{~mm}$. (E) Representative ECG tracing of TAC-operated ApoE ${ }^{-/-}$subjected to the physical stress protocol: (from left to right) during rest; the same mouse a week later showed ST elevation (red arrow) at rest; and following treadmill run ST elevation was further increased. The mouse died with remarkable signs of ST elevation and profound bradycardia (right). (F) Masson's trichrome staining of (left) the banded WT heart at 12 weeks after TAC, (middle) the TAC-operated ApoE ${ }^{-/-}$mouse that survived the physical stress (25 weeks after TAC), and (right) the TAC-operated ApoE-/- mouse that died during physical stress at 12 weeks after TAC. Scale bar: 1 mm. (C) Fractional shortening (FS) measured at the indicated time points after TAC $(n \geq 5)$. ${ }^{* *} P<0.01,{ }^{* * *} P<0.001,1$-way ANOVA. Representative images of 2-dimensional M-mode echocardiography of the LV of TAC-operated ApoE $\mathrm{E}^{-/-}$mice $(\mathbf{H})$ without infarct and (I) with infarct, showing the thin ventricular wall in the apex (yellow dotted lines).

coronary vasculature, specifically within $5 \mathrm{~mm}$ from the AV (Figure 1D). The lipid plaque composition followed a similar longitudinal profile (Figure 1E).

Based on the analysis of the LAD stenosis, we defined the proximal area of the LAD as a "hot spot" (Figure 1F). It is noteworthy to mention that the hot spot in this murine model of coronary lesions is similar to the one observed in human coronary arteries (19).

As expected, cholesterol levels in $\mathrm{ApoE}^{-/}$mice were significantly higher than those in WT mice (6) and were not different between sham- and TAC-operated $\mathrm{ApoE}^{-/-}$mice (Figure 1G), suggesting that the high pressure following aortic banding, in combination with moderate high cholesterol $(\sim 350 \mathrm{mg} / \mathrm{dl})$, is responsible for coronary plaque formation. The analysis of coronary lesions in the hearts of 34 TAC-operated $\mathrm{ApoE}^{-/-}$mice revealed that $59 \%$ developed LAD stenosis, 35\% showed early lesions without stenosis, and only $6 \%$ had no evidence of atherosclerosis (Figure $1 \mathrm{H}$ ). 
Evidence of $M I$ in $A p o E^{-/}$mice exposed to physical stress after TAC. By 10 weeks after TAC, more than $60 \%$ of $\mathrm{ApoE}^{-/-}$mice had died. Postmortem examination of some of these mice revealed areas of pale myocardium, suggestive of MI.

Next, ApoE ${ }^{-/-}$mice were physically stressed by treadmill running (once per week), starting at 8 weeks after TAC, when coronary lesions were established. During this protocol, echocardiographic analysis and ECG telemetry were performed (Figure $2 \mathrm{~A}$ ). After 25 weeks, $17 \%$ of the TAC-operated ApoE ${ }^{-1-}$ mice survived, $17 \%$ died before the physical stress protocol, and $66 \%$ died during the physical stress protocol (Figure 2B). The Kaplan-Meier curve in Figure $2 \mathrm{C}$ shows that $50 \%$ of the $\mathrm{ApoE}^{-/-}$mice died by week 14 and about $80 \%$ of the mice died by week 25 . Postmortem analysis of these mice also revealed pale myocardial areas suggestive of infarct (Figure 2D).

Eight TAC-operated ApoE ${ }^{-/-}$mice were implanted with radiotelemetry devices to measure single-lead ECG once per week, at rest, during, and 48 hours after physical exercise. ECG traces showed a clear ST elevation in 3 mice, and arrhythmic events in the remaining. The latter included accelerated idioventricular rhythm, which is associated with myocardial ischemia, and sudden profound bradycardia (sinus bradycardia and atrioventricular block). In the ECG depicted in Figure 2E, the ApoE ${ }^{-/-}$mouse at 9 weeks after TAC showed ST elevation at rest, which became more prominent during exercise, and the mouse died immediately after running with associated sudden bradycardia.

Masson's trichrome staining of banded hearts from mice that died with ST elevations revealed an extended cardiac fibrosis due to infarction damage. As controls, the Masson's trichrome staining was performed on the banded hearts of WT (Figure 2F, left) and surviving ApoE ${ }^{-/-}$(Figure 2F, middle) mice at the same time point after TAC, showing some degree of fibrosis, as expected in pressure-overloaded hearts, but substantially less than the infarcted $\mathrm{ApoE}^{-/-}$hearts (Figure 2F, right).

Finally, as expected fractional shortening was decreased following TAC (8-25 weeks, Figure 2G). Echocardiographic images showed left ventricular dilation, reduced function, and evident ventricular wall thinning in infarcted hearts (Figure $2 \mathrm{H}$ ) compared with noninfarcted hearts (Figure 2I) of TAC-operated ApoE $\mathrm{E}^{-/-}$mice.

Evidence of plaque disruption, thrombosis, and MI TAC-operated ApoE ${ }^{-/-}$mice. Hearts of TAC-operated $\mathrm{ApoE}^{-/-}$mice that died during the physical stress protocol were systematically sectioned from the AV to the apex and stained with H\&E. This first histological examination allowed us to identify points of plaque occlusion or disruption for further analysis. H\&E staining of sequential myocardial sections revealed 2 severe atherosclerotic lesions in the $\mathrm{LAD}$ at $2.95 \mathrm{~mm}$ from the $\mathrm{AV}$ (Figure $3 \mathrm{~A}$, image 4, asterisks, and Figure 3, B-G). Juxtaposed to the right plaque, in Figure 3A, inset i, there are noticeable "white" thrombi (arrowheads) and red blood cells confined within the narrow lumen. Next, we sought to determine whether plaque rupture and/or erosion caused the thrombotic event. Thus, myocardial sections, upstream and downstream to the one shown in image 4 (Figure 3A), were stained for CD41 to identify platelets aggregates, $\alpha$-smooth muscle actin ( $\alpha$ SMA) for SMC, and collagen I (Col I, marker of extracellular matrix) to define the fibrous cap of the plaque (Figure 3C). The immunofluorescent staining of the LAD showed a thin but continuous fibrous cap of the left plaque (Figure 3C, i) and 2 points of rupture in the fibrous cap of the right plaque, evidenced by the interrupted Col I staining (Figure 3C, ii). In Figure 3D, CD41 staining (red) of a consecutive section, confirmed the presence of a thrombus, which extended from the lumen to inside the ruptured plaque (Figure 3D, ii). These events are also depicted in the image in Figure 3E. It appears also clear that the thrombus is confined to the lumen in front of the left plaque (Figure 3, D and F, and relative $i$ images) with thin and undisrupted fibrous cap. Perhaps erosion might have occurred as the thrombus juxtaposes to the luminal surface of the left plaque.

In distal myocardial sections, the thrombus further extended into the ruptured plaque in intimate contact with the thrombogenic material (Figure 3, H and I as well as G and J). Moreover, CD31 staining (ECs) showed the lack of the endothelium on the right-ruptured plaque, while a positive staining can be observed in the upperleft part of the lumen (Figure 3I). Masson's trichrome staining of cross sections from the medium and apex of the heart revealed some perivascular and interstitial fibrosis but no signs of chronic MI (Figure 3K).

Another example of coronary plaque rupture is shown in Supplemental Figure 4. It is noteworthy to mention that platelet aggregation it is not present in proximal or distal myocardial sections to $2.95 \mathrm{~mm}$ from $\mathrm{AV}$, suggesting that the observed thrombus did not result from stagnant blood flow in the coronary vasculature. Masson's trichrome demonstrated minimal cardiac fibrosis, suggesting the onset of an acute MI due to plaque rupture (Supplemental Figure 4P). 
A
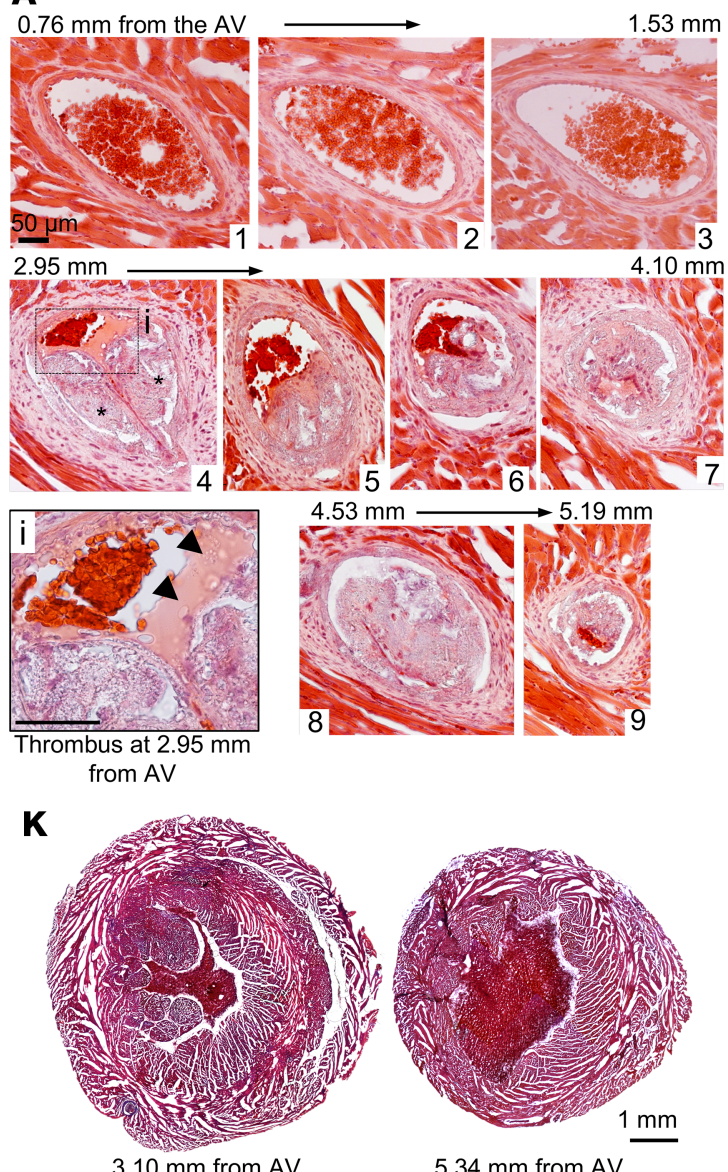

$3.10 \mathrm{~mm}$ from AV

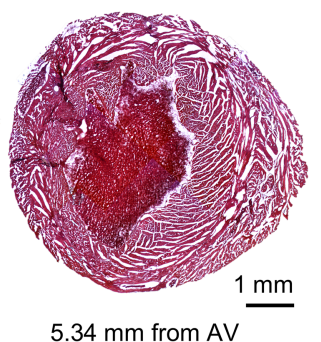

$5.34 \mathrm{~mm}$ from AV
B
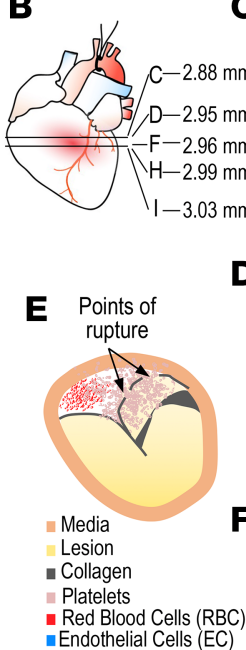

- Endothelial Cells (EC)

G Thinned buried

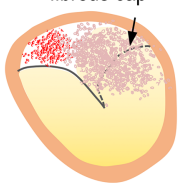

J

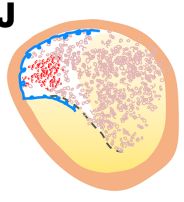

c

F

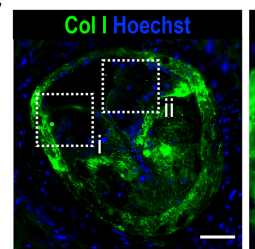

D
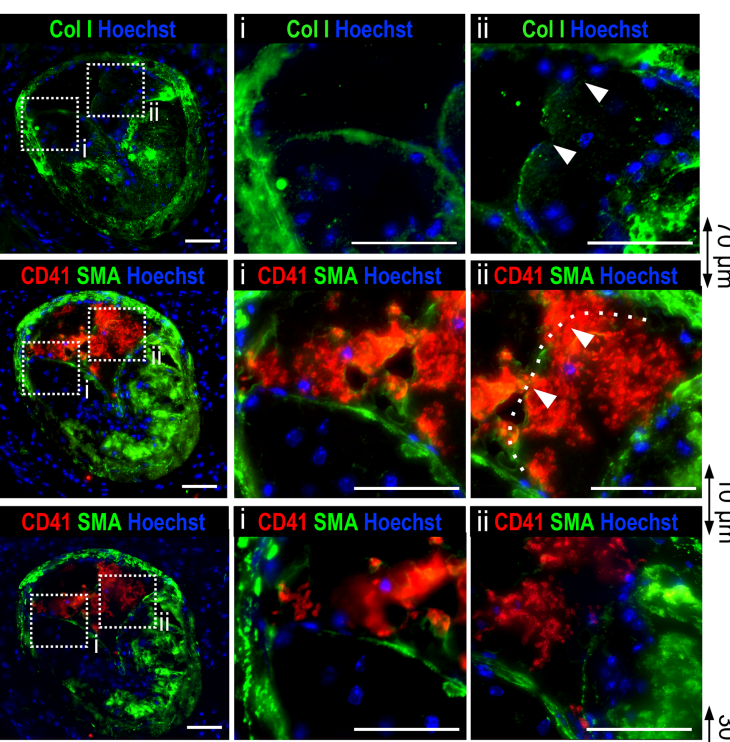

H
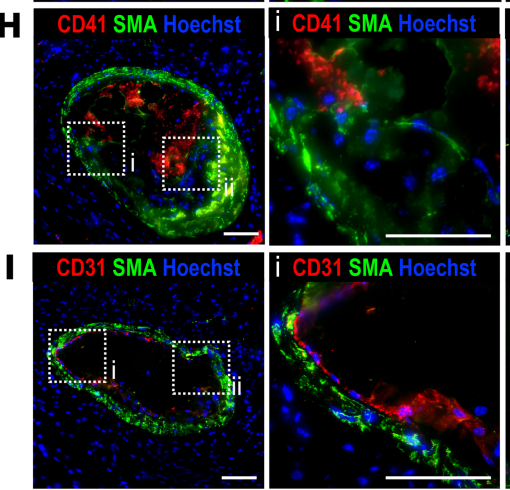

Figure 3. Coronary plaque rupture and thrombosis in the LAD of TAC-operated ApoE-/- mice. Sequential myocardial histology of TAC-operated ApoE ${ }^{-/}$ mice that died during physical stress protocol. (A) H\&E staining of myocardial sections showing the LAD. At 2.95 mm from the AV there are 2 major lesions and a thrombus (light pink) juxtaposed to the plaque on the right (asterisks, image 4, and i). (i) Magnified image of the thrombus with visible platelets (arrowheads) and red blood cells confined in a narrow lumen space. (B) Scheme of the localization of immunofluorescently stained myocardial sections proximal and distal to the thrombus shown in A, image 4. (C) IF staining of the LAD for collagen type I (Col I, green) and Hoechst (nuclei, blue), showing a thin and continuous fibrous cap on the left plaque (i) and a thin and discontinuous fibrous cap of the right plaque (ii). The 2 ruptured points are indicated by 2 arrowheads. (D) IF staining for CD41 (platelets, red), aSMA (smooth muscle cells, green), and Hoechst (blue) shows luminal thrombus juxtaposed to the left plaque (i). In ii, the thrombus is present in front of and within the right-ruptured plaque (arrowheads and dotted line). (E) Schematic representation of the IF staining in $\mathbf{D}$ and $\mathbf{F}$ of the ruptured plaque and thrombus in the LAD. (F) CD41, $\alpha$ SMA, and Hoechst IF staining of proximal consecutive section of the LAD, showing the same pattern of thrombus distribution in the lumen and within the ruptured plaque. (G) Schematic representation of the IF staining in $\mathbf{H}$ of the ruptured plaque and thrombus in the LAD. (H) Consecutive distal sections showing platelet infiltration and aggregation deeper into the plaque. Part of the right plaque detached from the arterial wall (dotted line). (I) IF staining for CD31 (EC) and $\alpha$ SMA of a consecutive distal section showing CD31-positive staining only in the upper left corner of the LAD, whereas is absent in the remaining $L A D$, where the ruptured plaque material and clots broke away from the wall. (J) Schematic representation of the IF staining in I of the disrupted endothelium in the LAD. (K) Masson's trichrome staining of the medium and apex of the heart at the indicated distance from the AV. Scale bars: $50 \mu \mathrm{m}$.

A significant cause of acute coronary syndrome is superficial plaque erosion (20). This event involves fibrous, rather than lipid-rich plaque, and disturbed flow may contribute to endothelial prothrombotic phenotype and apoptosis $(21,22)$.

$\mathrm{H} \& \mathrm{E}$ staining of sequential myocardial sections of a TAC-operated $\mathrm{ApoE}^{-/-}$mouse that died during the physical stress protocol showed the presence of a "white" thrombus at about $3 \mathrm{~mm}$ from the AV (Figure 4, A and B). Immunofluorescent staining of consecutive myocardial sections for CD41 corroborated this observation. Whereas at $2.57 \mathrm{~mm}$ from the AV - upstream the point of erosion no thrombus was observed (Figure 4, C-E), at about $3 \mathrm{~mm}$ from AV, CD41 staining evidenced the presence of thrombus in the lumen, adjacent to, but not inside the plaque (Figure 4, F and G). This observation was supported by Col I staining showing a thick fibrous cap, thus excluding the rupture of the plaque (Figure 4, H, I, and K). The lack of notable EC nuclei in Figure 4, H and I, suggested 
A

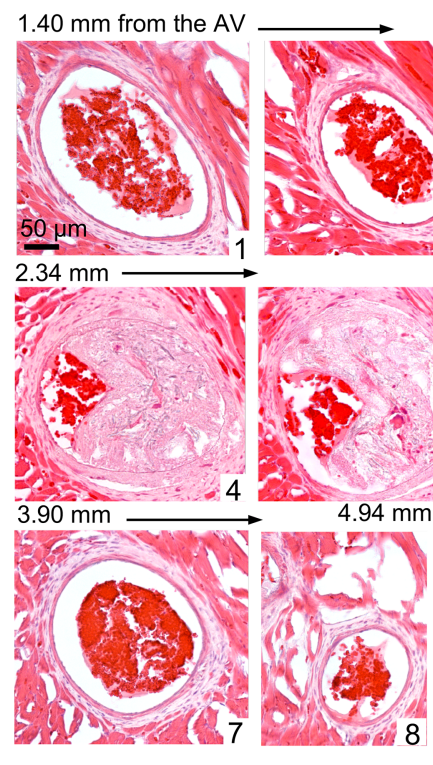

$\mathbf{L}$

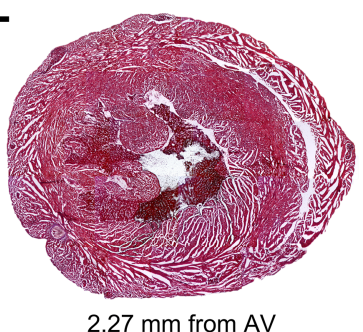

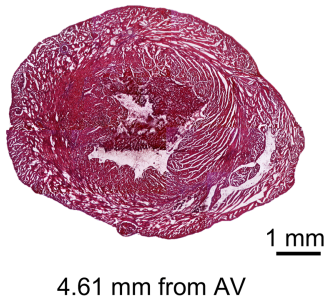

$.61 \mathrm{~mm}$ from AV
$2.08 \mathrm{~mm}$

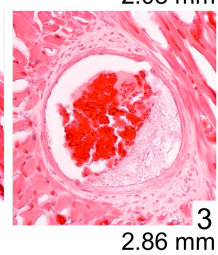

$2.86 \mathrm{~mm}$
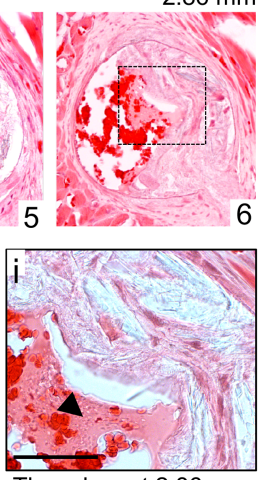

Thrombus at $2.86 \mathrm{~mm}$ from AV

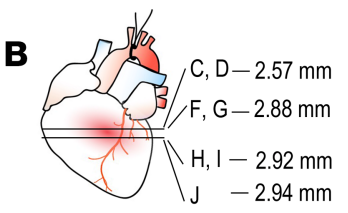

E

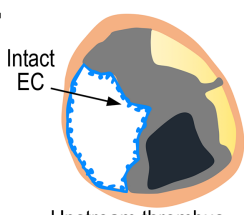

Upstream thrombus Downstream thrombus

Media

Lesion

- Collagen

Platelets

- Red Blood Cells (RBC)

- Endothelial Cells (EC)

- Necrotic Core
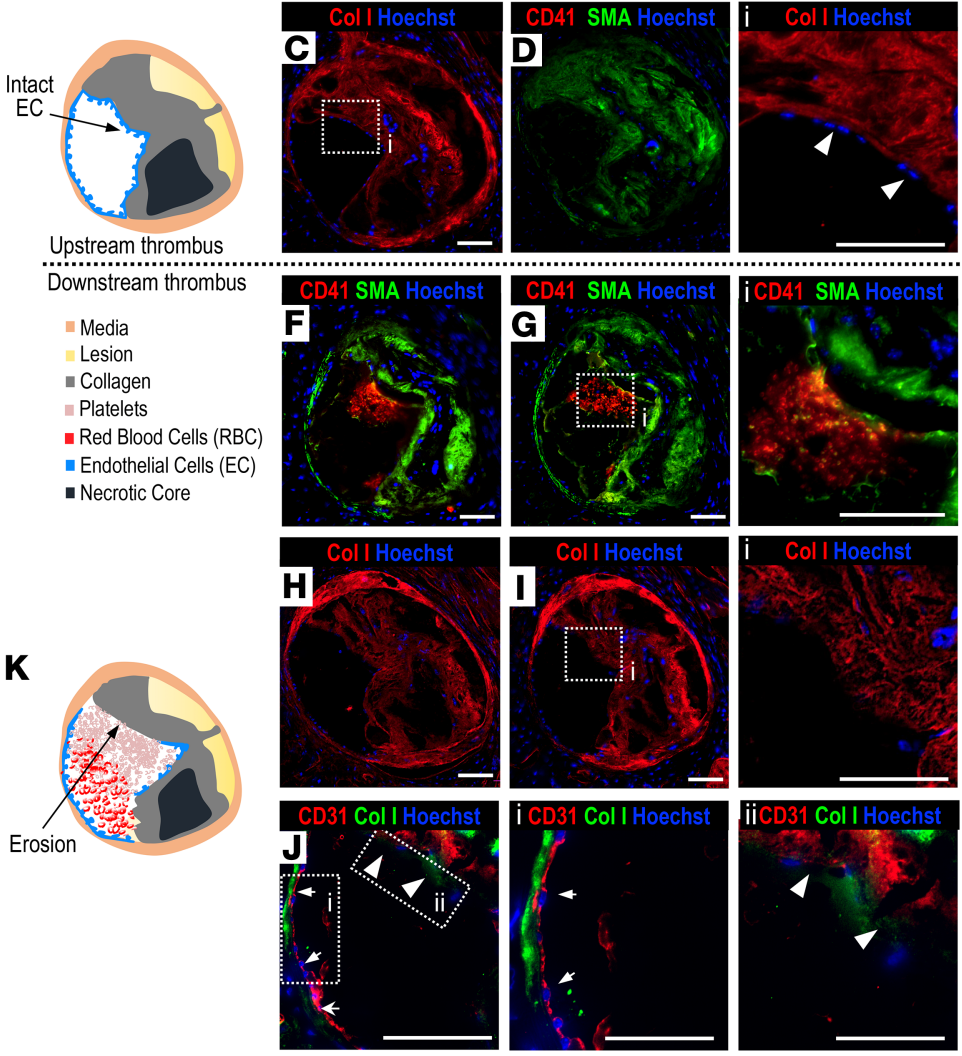

Figure 4. Coronary plaque erosion and thrombosis in ApoE-/- mice after TAC. (A) H\&E staining of sequential myocardial sections of ApoE $\mathrm{H}^{-/-}$mice that died during the physical stress protocol. At $2.86 \mathrm{~mm}$ from AV (image 6), it is noticeable that the thrombus (light pink) is in contact with the coronary plaque of the LAD. Arrowhead indicates visible platelets. Scale bar: $50 \mu \mathrm{m}$. (B) Scheme of the localization of immunofluorescently labeled heart sections proximal and distal to the section at $2.86 \mathrm{~mm}$ from the AV. (C and D) IF staining of section proximal to erosion for Col I, CD41, $\alpha$ SMA, and Hoechst showed the absence of platelet aggregation. Hoechst staining shows the presence of elongated endothelial cell nuclei (C, i, arrowheads). (E) Schematic representation of the IF staining upstream the erosion shown in C and D. (F-I) IF for Col I, CD41, $\alpha$ SMA, and Hoechst of myocardial sections displaying the thrombus in the lumen, adjacent to but not inside the plaque (G, i). The fibrous cap (Col I and $\alpha$ SMA staining) adjacent to the thrombus is very thick and lacks of endothelial cell nuclei (I, i). (J) The staining for CD31 shows the presence of endothelial cells on the left side of the LAD free from platelet aggregates (J, i, arrows) but the absence of endothelial cells on the right side of the plaque in contact with the thrombus (J, ii, arrowheads). (K) Schematic representation of the IF staining of the eroded plaque with the thrombus in the LAD shown in $\mathbf{H}$ and J. Scale bars: $50 \mu \mathrm{m}$. (L) Masson's trichrome staining of the medium and apex of the heart at the indicated distance from the AV. Scale bar: 1 mm.

erosion of the endothelium. CD31 staining confirmed the absence of the endothelium on the plaque in contact with the thrombus (Figure 4J, ii, arrowheads), whereas positive CD31 staining was present on the opposite site of the LAD (Figure 4J, i, arrows, and $\mathrm{K}$ ), where the thrombus was absent. These data suggest that coronary plaque erosion triggered the formation of thrombus; contributing to MI. Masson's trichrome staining of the heart with minimal fibrosis suggests the onset of an acute MI due to erosion rather than chronic infarct (Figure $4 \mathrm{~L}$ ).

Evidence of MI caused by coronary occlusion in TAC-operated ApoE $E^{-/}$mice. Histological analysis of the heart showed coronary stenosis atherosclerosis and no evidence of plaque disruption and thrombosis (Figure 5A). Specifically, the occlusion of LAD and/or its branches was evident (i.e., diagonal and marginal arteries). H\&E, Masson's trichrome, and Oil Red O staining of myocardial sections from the base to apex revealed the occlusion of the distal LAD and diagonal arteries (Figure 5, B-D). These advanced plaques were filled with cholesterol crystals (Figure 5C, i and ii) and lipids (Figure 5D). Five of the six -TAC-operated ApoE ${ }^{-/-}$mice presenting with coronary occlusions were also evaluated with ECG telemetry during the physical stress protocol. 
A
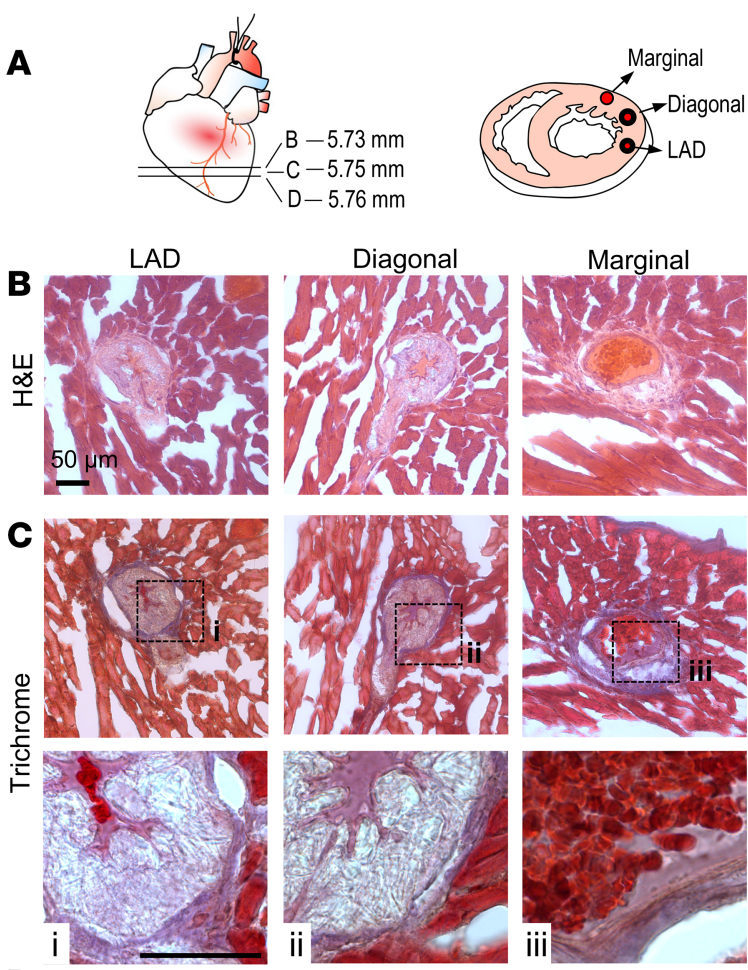

D

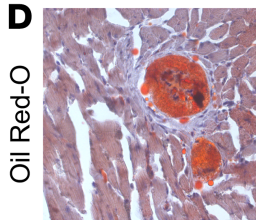

Diagonal
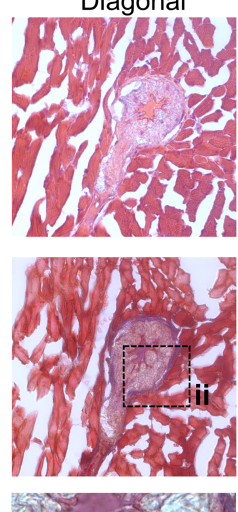
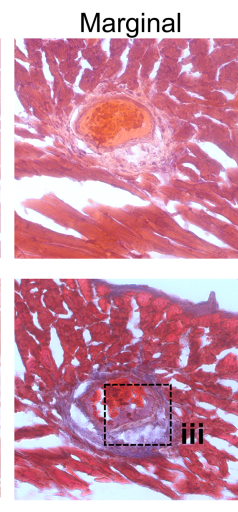

$\mathbf{E}$

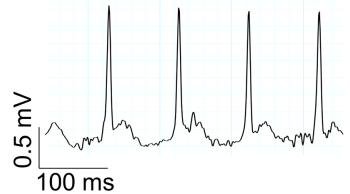

C. 11 wks post-TAC after $2 \min 6 \mathrm{sec}$ of run with wider ST elevation

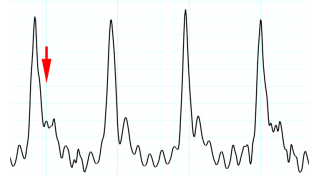

b. 11 wks post-TAC after $46 \mathrm{sec}$ of run

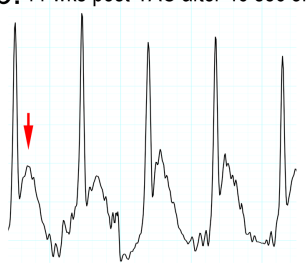

Transition from ST elevation to d. idioventricular rhythm at $2 \mathrm{~min} 10 \mathrm{sec}$

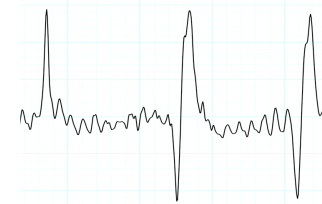

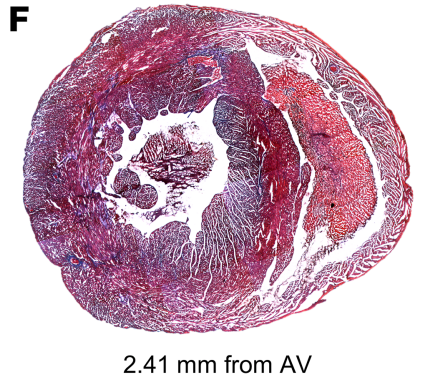

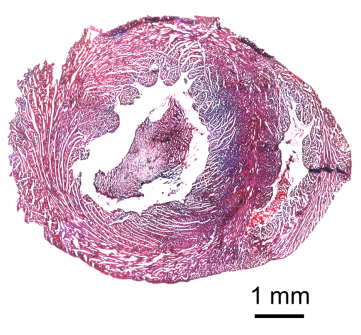

$5.91 \mathrm{~mm}$ from AV

Figure 5. Coronary occlusions without thrombus in epicardial coronary arteries of ApoE-/- mice after TAC affected by MI. (A) Scheme of the localization of heart sections stained with H\&E, Masson's trichrome, and Oil Red O in the apex (left), and image of the occluded coronary arteries (right). (B) H\&E, (C) Masson's trichrome, and (D) Oil Red $\mathrm{O}$ staining of distal coronary arteries, showing remarkable atherosclerotic occlusion, and likely vasospasm, of the LAD and diagonal arteries, but not of the marginal artery, at $5.73 \mathrm{~mm}$ from AV. Scale bars: $50 \mu \mathrm{m}$. (E) ECC tracing measured at 11 weeks after TAC in ApoE ${ }^{-/-}$ mouse at rest (top left). ST elevation occurred after 46 seconds of running (top right), and it became wider after 2 minutes of running (bottom left). The mouse died after a transition from sinus tachycardia with ST elevation to an idioventricular rhythm (bottom right). (F) Masson's trichrome staining of the medium and apex of the heart at the indicated distance from the AV. Scale bar: $1 \mathrm{~mm}$.

Two mice showed ST elevation, and three mice manifested an accelerated idioventricular rhythm, suggestive of myocardial ischemia.

Figure 5E shows the ECG changes of an $\mathrm{ApoE}^{-/-}$mouse at 11 weeks after TAC at rest and during the run on the treadmill. ST elevation was evident already at 46 seconds of running (Figure 5E). After 2 minutes of running, the QRS complex became wider, finally leading to an accelerated idioventricular rhythm and death. In addition to the coronary occlusion, coronary spasm could also have contributed to the myocardial event.

Interestingly, Masson's trichrome staining of the hearts of all 6 mice that died during the physical stress protocol and presenting with coronary occlusion showed evidence of extended fibrosis (Figure 5F), suggesting chronic MIs, which might have contributed to stress-induced death from ischemic cardiomyopathy.

Evidence of coronary embolism in TAC-operated Apo $\mathrm{E}^{-/-}$mice. Coronary artery embolism is a rare nonatherosclerotic cause of acute MI, attributed mainly to atrial fibrillation, followed by dilated cardiomyopathy and valvular heart disease (23). In most cases, coronary artery embolism involves the distal LAD, causing transmural MI (24). Interestingly, we found a few cases of abrupt occlusion of coronary arteries without significant atherosclerosis. H\&E staining of myocardial sections from the base to the apex clearly showed the absence of atherosclerotic plaques, with abrupt interruption of blood flow in the distal LAD at approximately $6 \mathrm{~mm}$ from the AV (Figure 6, A and B). It is possible to notice red blood cells in image 7 and the thrombotic occlusion in the distal image 8 of Figure 6A. CD41 immunofluorescent staining displayed platelet aggregation occluding the entire lumen and disrupting the continuity of the endothelium in some 
A

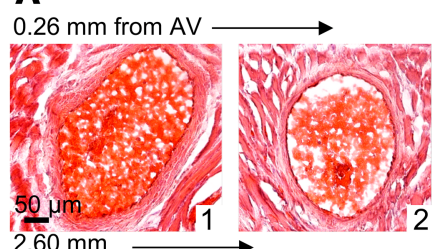

$2.60 \mathrm{~mm}$
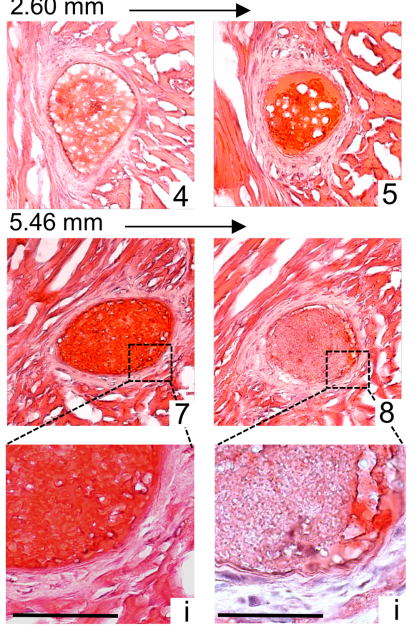
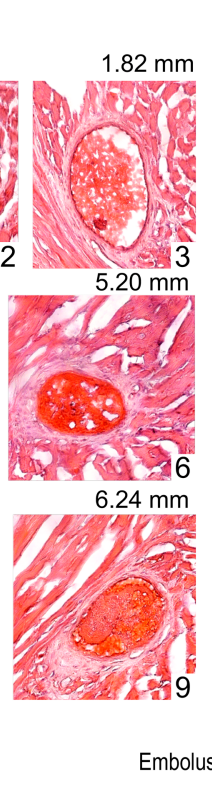

$$
\begin{aligned}
& \text { - Platelets } \\
& \text { Red Blood Cells (RBC) } \\
& \text { - Endothelial Cells (EC) }
\end{aligned}
$$$$
\text { Media }
$$

B
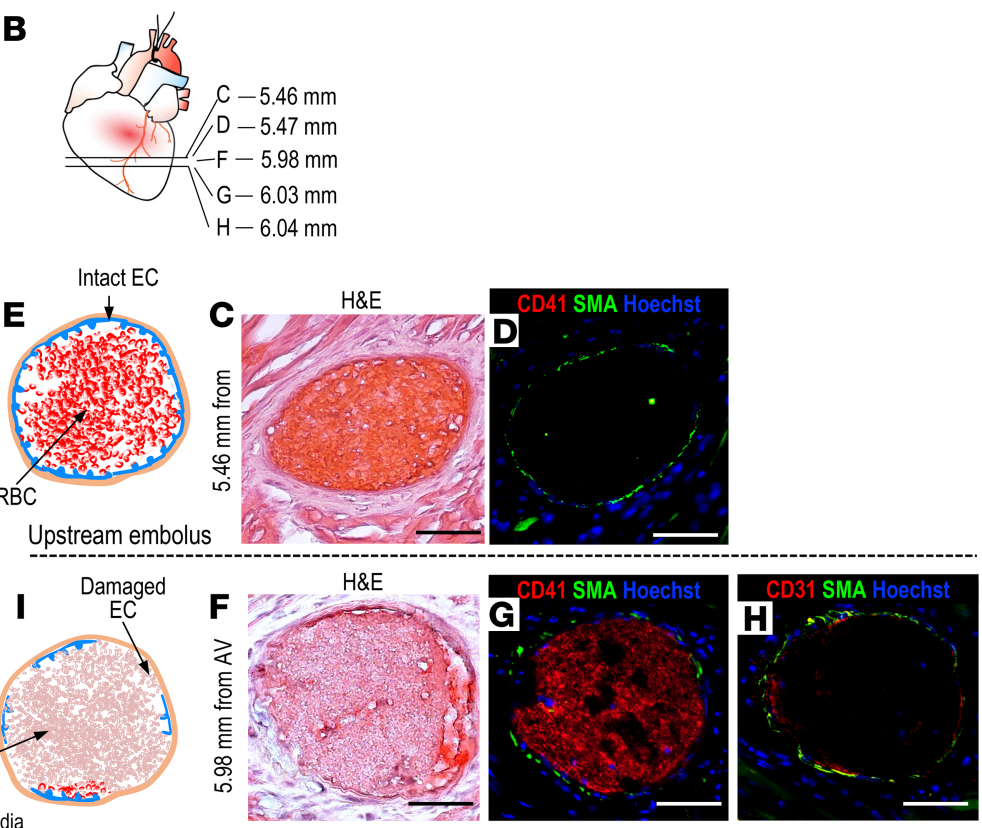

J

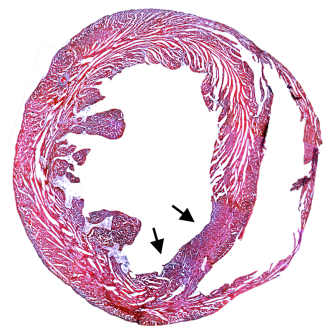

$3.73 \mathrm{~mm}$ from AV

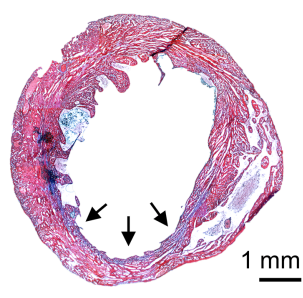

$5.67 \mathrm{~mm}$ from AV

Figure 6. Coronary artery embolism in the distal LAD of TAC-operated ApoE-/- mice. (A) H\&E staining of myocardial sections of a ApoE ${ }^{-/-}$mouse that died during the physical stress protocol. Scale bars: $50 \mu \mathrm{m}$. (B) Scheme of the localization of immunofluorescently labeled heart sections proximal and distal to the section with thrombus at $5.98 \mathrm{~mm}$ from the AV, shown in A, image 8. (C and D) H\&E and IF staining of consecutive myocardial sections upstream the embolus. CD41 staining is negative, confirming the absence of thrombus at about $0.5 \mathrm{~mm}$ upstream the myocardial section in $\mathbf{A}$, image 8 . (E) Schematic representation of the LAD staining in $\mathbf{C}$ and $\mathbf{D}$. (F-H) Embolus was detected by H\&E and IF staining for Col I, CD41, CD31, $\alpha$ SMA, and Hoechst. The endothelium appears partially damaged, most likely by the thrombus, since there are no evident lesions. (I) Scheme of the immunofluorescently labeled LAD shown in F-H. Scale bars: $50 \mu \mathrm{m}$. (J) Masson's trichrome staining of the medium and apex of the heart at the indicated distance from the AV. Arrows indicate chronic infarcted tissue. Scale bar: $1 \mathrm{~mm}$.

areas (Figure 6, F-I) but not upstream the thrombus (Figure 6, C-E). In Figure 6J, Masson's trichrome staining revealed endocardial fibrosis and left ventricular dilation, which may have contributed to the formation of thrombosis that caused the coronary embolism. Because of the absence of significant atherosclerosis in this mouse and the presence of thrombus creating an abrupt occlusion of the distal LAD, we classified this case as coronary embolism.

Causes of $M I$ in TAC-operated ApoE $E^{-1-}$ mice subjected to the physical stress protocol. In addition to systematic histological analysis, ECG, and echocardiographic measurements, we also performed electron microscopy on the pale myocardial tissue from TAC-operated $\mathrm{ApoE}^{-/-}$mice that died during physical stress protocol. As control hearts, we used $\mathrm{ApoE}^{-/-}$(with no sign of $\mathrm{MI}$ ) and C57BL/6 mice at the same time point after TAC. Mitochondria in control hearts had preserved morphology, with intact double membrane, compact and organized cristae, and homogeneous dense matrix (Supplemental Figure 5A). On the contrary, infarcted hearts showed the typical mitochondrial ultrastructural hallmarks of irreversible injury, including cell swelling, loss of mitochondrial double membrane, and fragmented reticular mitochondria, with distended and disorganized cristae and dishomogeneous matrix (Supplemental Figure 5, B-D), supporting the occurrence of MI.

Based on all this evidence, we found that $74 \%$ of the TAC-operated ApoE ${ }^{-/-}$mice that died during the physical stress protocol showed evidence of MI (Figure 7A). In 17\% of the cases we were not able to collect the hearts because the carcasses were removed from the cages. The remaining $9 \%$ of the mice had no evidence of coronary atherosclerosis and MI. 
A

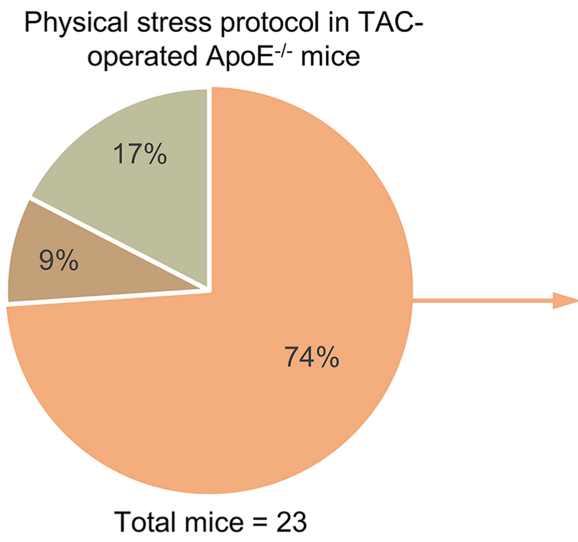

Evidence of MI (TTC or ECG + histology)

No evidence of plaque and $\mathrm{Ml}$

Hearts not collected
B Causes of MI

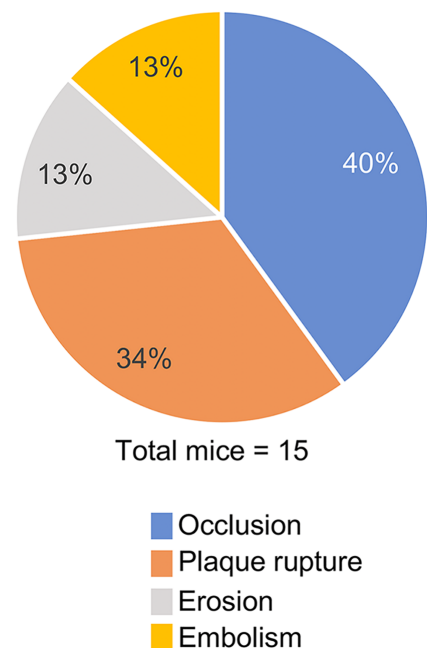

Figure 7. Causes of $\mathrm{MI}$ in TAC-operated $\mathrm{ApoE}^{-/-}$mice subjected to physical stress. (A) Pie diagram of the TAC-operated $A \mathrm{poE}^{-/-}$mice that died during the physical stress protocol: $74 \%$ of the mice presented evidence of $\mathrm{MI}$, determined by TTC staining or ECG and histological analysis; $9 \%$ did not show evidence of coronary plaque and MI; and $17 \%$ of the mice were discarded by the technicians of the Research Animal Resource Center and therefore the heart were not collected ( $n=23 \mathrm{TAC}$-operated $\mathrm{ApoE}^{-/-}$mice). (B) Pie diagram illustrating the causes of $\mathrm{Ml}$ of the $74 \%$ of animals that died during physical stress: $40 \%$ of the mice showed coronary occlusion; $34 \%$ had evidence of plaque rupture; $13 \%$ had evidence of plaque erosion; and $13 \%$ had evidence coronary embolism ( $n=15 \mathrm{TAC}^{-o p e r a t e d ~} \mathrm{ApoE}^{-1-}$ mice).

We were able to define and quantify the causes of MI as follows: $47 \%$ of the mice presented clear evidence of coronary thrombosis, due to plaque rupture (34\%) and plaque erosion (13\%), and $40 \%$ of the mice showed plaque occlusion, with evidence of chronic myocardial fibrosis (Figure 7B). Two mice had abrupt occlusion of coronary arteries by thrombus with no evidence of atherosclerosis, which was therefore classified as coronary embolism (13\%).

\section{Discussion}

For long time, establishing a mouse model of coronary atherosclerosis and its progression has been the "holy grail" in atherosclerosis research. ApoE $\mathrm{E}^{-/-}$and $\mathrm{Ldll}^{-/}$mice have been extensively used to study the pathogenesis of atherosclerosis (25) and provided great insights into the mechanisms of human disease. Studies in mice identified different genes involved in atherogenesis, and some of them have been confirmed in human pathology, including genes involved in triglyceride-rich lipoprotein metabolism $(26,27)$ and PCSK9 (protein convertase subtilisin/kexin type 9) $(28,29)$. Mouse models have proven to be very valuable to study disease mechanisms; however, many limitations exist that need to be taken into account, especially considering the challenges in translating the biology of atherosclerosis.

To develop atherosclerotic lesions, mice require cholesterol levels $(\sim 2000 \mathrm{mg})$ that far exceed those commonly present in humans (9). More importantly, murine atherosclerosis develops in the aorta and its proximal branches, whereas coronary arteries are typically spared. The hemodynamics of the aorta and coronary arteries, as well as the origins of their SMCs, differ dramatically. SMCs of coronary arteries originate from the proepicardial organ, while the ones of descending aorta arise from somatic precursors $(30$, $31)$. Furthermore, plaque rupture, erosion, and thrombus formation, which are the most critical complications of human atherosclerosis, are not present in current models $(25,32,33)$. Whereas patients are treated at late stage of the disease when symptoms arise, mechanisms studied in mice focus on the development of atherosclerosis more than its progression. The difficulties in translating some of the studies in atherosclerosis has been ascribed, at least in part, to the aforementioned limitations of current mouse models $(4,9,34$, $35)$. To date, few attempts have been made to "model" coronary atherosclerosis and its sequelae in mice $(10,11)$, without realistic success.

Our study identifies the experimental conditions to induce the formation of coronary atherosclerosis and myocardial events by combining moderated hypercholesterolemia $\left(\mathrm{ApoE}^{-/-}\right.$mice on chow diet) with 
increased afterload (TAC surgery), two major risk factors for CAD (36-39) and its complications. We have systematically characterized the topography, the magnitude, and lipid composition of coronary plaques, in particular of the LAD (Figure 1). The major finding of our study is the formation of coronary lesions in mice exposed to specific pathological conditions (high cholesterol and pressure), which can progress over time and lead to MI under physical stress conditions (tread mill run), mainly due to coronary disruption and thrombosis and coronary occlusion (Figure 7). Physical exertion (40) and emotional stress may act as external triggers for acute $\mathrm{MI}$ (41). Similarly, in TAC-operated $\mathrm{ApoE}^{-/-}$mice, physical stress induced myocardial events, in most of the mice (74\%), during or within 48 hours from the treadmill run.

Most emphasis has been placed on the role of endothelium and macrophages in the initiation and progression of early atherosclerosis. However, human autopsy studies $(42,43)$ and in vivo data suggest an important role of SMCs in the initial phase of atherosclerosis. As macrophages, SMCs are known to express a variety of cholesterol uptake receptors, including LDL receptor (44), CD36 (45), and VLDL receptor (46), and, therefore, can form foam-like cells and participate in atherogenesis. SMCs are also able to express adhesion molecules and retain inflammatory cells in the vascular wall. In early coronary lesions of $\mathrm{ApoE}^{-/-}$mice at 8 weeks after TAC, in addition to macrophages and ECs, lipid accumulation was found in SMCs of the media (Figure 1), suggesting an active role of coronary SMCs in the development of atherosclerosis in mice. Interestingly, as in humans, early coronary atherosclerosis was tightly associated with neointimal proliferation.

$\mathrm{ApoE}^{-/-}$mice on high-fat diet showed evidence of buried plaques in the innominate artery $(47,48)$ but no signs of the coronary lesions and disruption $(49,50)$, which is the cause of about $75 \%$ of coronary thrombi formation in humans $(51,52)$. Our study is the first to our knowledge demonstrating coronary plaque formation and progression toward disruption (rupture and/or erosion) and occlusion, which were accountable for $87 \%$ of the MIs.

It is generally accepted that macrophages play an important role in the progression of atherosclerosis, by contributing as an important source of enzymes catabolizing collagen, a key component of the fibrous cap of the plaque $(9,53,54)$. Interestingly, immunofluorescent staining (CD68 for macrophages and $\alpha$ SMA for SMCs) evidenced a robust accumulation of macrophages in the ruptured plaque and specifically adjacent to the points of break of the thin fibrous cap (Supplemental Figure 4E). We are aware that these lineage markers for SMC ( $\alpha$ SMA) and macrophages (CD68) are promiscuous (55), as some cells are both, CD68 and $\alpha$ SMA positive. The aim of these stainings was not to quantitatively distinguish between these 2 populations but to show the presence of macrophages in the ruptured plaques. Interestingly, in Supplemental Figure 4, it is noticeable that the majority of CD68 and $\alpha$ SMA staining does not colocalize, but only a minor population is double positive. Therefore, even considering these limitations, our data support the abundance of macrophages in the ruptured plaque, in correspondence to the thinning of the fibrous cap and breaking points.

In $40 \%$ of the mice studied, we found evidence of coronary ischemia based on ECG monitoring that revealed ST elevation in some cases and arrhythmic events, such as accelerated idioventricular rhythm, in others. Indeed, we found stenosis and occlusions of the LAD arteries as well as other major coronary arteries (diagonal and marginal) in our mouse model. It is possible that, under physical stress, demand-related ischemia due to $\mathrm{CAD}$ and prior infarctions resulted in demise. Some mice also demonstrated acute MI potentially triggered by increased shear stress from the exercise. Prolonged ischemia has been shown to induce vasoconstriction in animals and humans (56-58).

Finally, in a small percentage of the infarcted hearts, we found no evidence of coronary atherosclerosis with an abrupt interruption of blood flow by thrombus (Figure 6). In humans, coronary artery embolism occurs at low frequency and is commonly caused by atrial fibrillation $(23,59)$. Interestingly, ApoE ${ }^{-/-}$mice following TAC presented a low rate of coronary embolism, and we did not observe atrial fibrillation in our mice. We do not know the origin of the embolus, but it is possible that the left ventricular dysfunction in our model contributed to formation of left ventricular thrombus.

Interestingly, when the same transstenotic gradient was applied to chow diet-fed Ldlr-/- mice, known to have a milder increase in cholesterol levels than $\mathrm{ApoE}^{-/-}$mice (60), coronary lesions developed at a later time point (Supplemental Figure 6), suggesting a direct correlation between cholesterol levels and coronary atherosclerosis in this mouse model of CAD. Lipid content, plaque distribution, and the magnitude of stenosis was similar between the 2 groups of mice. TAC-operated $\mathrm{Ldlr}^{-{ }^{-}}$mice that died during the physical stress protocol showed evidence of MI due to coronary occlusions (Supplemental Figure 7). 
Although further characterization is necessary to define the causes of MI, the finding that afterload leads to the development of coronary lesions in $\mathrm{Ldlr}^{-1-}$ mice is relevant, because this pathological model of CAD can be applied to complex genetically modified mice by using PCSK9 AAV technology (61).

In conclusion, the coexistence of high cholesterol and increased afterload can induce the formation of coronary lesions in approximately $95 \%$ of $\mathrm{ApoE}^{-/-}$mice and leads to plaque disruption and occlusion in more than $70 \%$ of the mice (Figure 7). This pathological phenotype recapitulates major complications of human coronary atherosclerosis, specifically the formation in mice of coronary lesions that can progress to disruption, leading to thrombosis or occlusion, ultimately causing myocardial events.

This study presents for the first time to our knowledge a reliable tool to investigate not only the pathogenesis of coronary atherosclerosis and its progression in $\mathrm{ApoE}^{-/-}$and $\mathrm{Ldlr}^{-/}$mice, but also potential therapeutic targets.

\section{Methods}

Minimally invasive TAC. WT C57BL/6J, $\mathrm{ApoE}^{-/-}$, and $\mathrm{Ldlr}^{-/-}$male mice (11-14 weeks of age; body weight 25-27 g) were subjected to TAC or sham operation (62). Briefly, mice were anesthetized using a single i.p. injection of ketamine $(100 \mathrm{mg} / \mathrm{kg})$ and xylazine $(5 \mathrm{mg} / \mathrm{kg})$. A topical depilatory was applied to the chest, and the area was cleaned with betadine and alcohol. A horizontal incision $0.5-0.8 \mathrm{~cm}$ in length was made at the second intercostal space. After retracting the thymus, the aortic arch was visualized with a dissecting scope (Zeiss Discovery.V8 Stereo) at low magnification. A 7-0 nylon ligature was tied between the innominate and left common carotid arteries with an overlying 28 -gauge needle, which was then rapidly removed, leaving a discrete region of stenosis. In sham-operated animals, the ligature was just tied loosely around the aorta.

Transstenotic gradient was measured in TAC-operated $\mathrm{ApoE}^{-/-}$mice subjected to a 28-gauge needle ligation by using Telemetry catheters (HD-X10; Data Sciences International). Briefly, 1 day after TAC, mice were anesthetized using a single i.p. injection of ketamine $(100 \mathrm{mg} / \mathrm{kg})$ and xylazine $(5 \mathrm{mg} / \mathrm{kg})$. A gel-filled catheter connected to a blood pressure transducer was inserted first into the right carotid (high pressure) and secured with a 7-0 silk suture. The transducer, which communicates with the receiver wirelessly, recorded blood pressure continuously using the Ponemah Physiology Platform (Data Sciences International). Values were recorded for 10 minutes at 1-minute intervals, each reading representing the average of blood pressure during 10-second sampling period. The catheter was then inserted into the left common carotid artery and secured with a 7-0 silk suture. Blood pressure was recorded for 10 minutes, and the animals sacrificed afterward. The transaortic gradient was expressed as the difference in blood pressure (systolic and mean) upstream and downstream of the banding.

Cholesterol levels. Total cholesterol was measured in plasma from sham- or TAC-operated WT and ApoE ${ }^{-/-}$mice, using a Cholesterol E kit (WAKO Life Science) according to the manufacturer's instructions. Briefly, whole blood was collected in EDTA via cardiac puncture. Plasma were obtained by centrifugation at $1,000 \mathrm{~g}$ for 15 minutes at $4^{\circ} \mathrm{C}$.

Stress-induced MI protocol. Starting from 8 weeks after TAC, ApoE $\mathrm{E}^{-/-}$mice were placed on the treadmill (Columbus Instrument) to run for $4 \mathrm{~min} / \mathrm{d}$, once a week, for 12 weeks, at a maximal sustained running speed of $13-14 \mathrm{~m} / \mathrm{min}$ with $15^{\circ}$ of inclination. Because this test is a form of forced exercise, when the mice sometimes stopped for short periods, they were gently tapped or lifted to encourage resumption of running. A minimal electric shock was also applied in order to encourage the run on the treadmill.

Radiotelemetry for electrocardiographic analysis. ECGs in conscious mice were measured using telemetry transmitters (ETA-F10 transmitter; Data Sciences International). Briefly, the transmitter device, which communicates with the receiver wirelessly, was inserted subcutaneously through a dorsal incision in the abdomen of the animal, and the 2 ECG electrodes were placed subcutaneously along the superior right pectoral region (negative lead) and the left caudal rib position (positive lead) to approximate lead II of the Einthoven surface ECG. Mice were housed individually in a temperature- and humidity-controlled facility and maintained under a 12-hour dark and 12-hour light cycle. They were allowed to recover for 7 days after surgery. ECG traces were continuously recorded at baseline, during the stress-induced MI protocol and during the following 48 hours, using LabChart software (AD Instruments).

Echocardiographic analysis. Cardiac dimensions and function were analyzed by transthoracic echocardiography using a Vevo 770 Imaging System (VisualSonics) as previously described (62). Mice were lightly anesthetized with inhaled isoflurane $\left(0.2 \%\right.$ in $\left.\mathrm{O}_{2}\right)$. Left ventricle M-mode was used, all measurements 
were obtained from 3-6 consecutive cardiac cycles, and the average values were used for analysis. Left ventricle end-diastolic (LVDd) and end-systolic (LVDs) dimensions were measured from the M-mode traces, and fractional shortening was calculated as follows: [(LVDd - LVDs)/LVDd]. Diastolic measurements were taken at the point of maximum cavity dimension and systolic measurements were made at the point of minimum cavity dimension, using the leading-edge method of the American Society of Echocardiography (63).

Oil Red $O$ staining. To assess lipid deposition in the coronary arteries, frozen heart sections were stained with Oil Red O (MilliporeSigma) as previously described (64). Briefly, heart cryosections were washed in PBS, stained with $0.2 \%$ Oil Red O in $60 \%$ isopropanol for 20 minutes, and washed with $60 \%$ isopropanol. After counterstaining performed with Mayer's hematoxylin, sections were washed and coverslipped with aqueous mounting medium. Images were acquired by using a Zeiss Axio Observer.Z1 microscope, and the area of Oil Red O staining was calculated by using ImagePro (Media Cybernetics). Coronary stenosis was calculated by measuring the area of the lumen $\left(\mathrm{A}_{\mathrm{L}}\right)$ and the internal elastic lamina $\left(\mathrm{A}_{\mathrm{IEL}}\right)$; the percentage of stenosis was calculated as follows: $\left(\mathrm{A}_{\mathrm{IEL}}-\mathrm{A}_{\mathrm{L}}\right) / \mathrm{A}_{\mathrm{IEL}} \times 100$. Oil Red $\mathrm{O}$ percentage in the plaque stenosis was calculated as the ratio of positive Oil Red $\mathrm{O}$ staining area to the percentage plaque area.

Masson's trichrome staining. Myocardial fibrosis was assessed in TAC-operated WT and ApoE ${ }^{-1-}$ mice by using Masson's trichrome stain kit (Polysciences Inc.) according to the manufacturer's instructions.

Histology and immunostaining. Mouse hearts were collected no more than 1 hour postmortem without perfusion and fixed with $4 \%$ paraformaldehyde (PFA) overnight at $4^{\circ} \mathrm{C}$. The following day, $4 \%$ PFA was replaced by $30 \%$ sucrose and left overnight at $4^{\circ} \mathrm{C}$ on rotation. The hearts were divided into 3 parts (base, center, and apex), submerged overnight with OCT/sucrose $30 \%$ (1:1 ratio), and consequently embedded in OCT. The whole heart was sectioned, with sets of 10 consecutive sections of $10 \mu \mathrm{m}$ collected every $30 \mu \mathrm{m}$. Heart sections every $260 \mu \mathrm{m}$ were routinely stained with $\mathrm{H} \& \mathrm{E}$ and coverslipped with aqueous mounting medium. For immunofluorescence analysis, the heart sections were permeabilized in $0.5 \%$ Triton X-100 in a solution of $5 \%$ BSA $5 \%$, followed by blocking in BSA $5 \%$ (45 minutes at room temperature). Next, the heart sections were incubated overnight at $4^{\circ} \mathrm{C}$ with anti-CD41 antibody (1:100, 553897; BD Pharmingen), anti- $\alpha$ smooth muscle actin antibody (1:200, 180186; Life Technologies), anti-CD31 (1:100, 553370; BD Biosciences), anti-CD68 antibody (1:200, MCA1957; Bio-Rad), and anti-collagen I antibody (1:200, ab43710; Abcam) in 5\% BSA. After 3 washes in PBS, secondary antibodies Alexa Fluor 568-conjugated donkey anti-mouse IgG (1:200, ab34710; Abcam), Alexa Fluor 647-conjugated goat anti-rat IgG (1:500, A21247, Thermo Fisher Scientific), and Cy5-labeled donkey anti-rabbit IgG (1:500, 711-175-152; Jackson ImmunoResearch) were added for 1 hour at room temperature. Nuclei were counterstained with Hoechst 33258 (1:10,000, 382061; Calbiochem). Immunofluorescence images of heart sections were captured in a $Z$-stack with $0.48-\mu \mathrm{m}$ steps using a Zeiss Axio Observer.Z1 microscope followed by deconvolution using the ZEN pro 2012 software (Zeiss).

Electron microscopy. A 1-mm thin tissue was collected from the anterior wall of the left 195 ventricle, showing a pale area. We used hearts of $\mathrm{ApoE}^{-/-} \mathrm{TAC}$-operated mice in which ECG traces showed ST elevation and control hearts from C57BL/6 mice at the same time points after TAC. Cardiac left ventricle tissues were fixed with a modified Karnovsky's fix (2.5\% glutaraldehyde, $4 \%$ PFA, and $0.02 \%$ picric acid in $0.1 \mathrm{M}$ sodium cacodylate buffer at $\mathrm{pH} 7.2$ ) and post-fixed in $1 \% \mathrm{OsO}_{4}$ and $1.5 \%$ $\mathrm{K}_{3}\left[\mathrm{Fe}(\mathrm{CN})_{6}\right]$. After dehydration, samples were embedded in an Epon analog resin. Ultrathin sections were cut using a Diatome diamond knife (Diatome) on a Leica Ultracut S ultramicrotome (Leica). Sections were collected on copper grids and stained with uranyl acetate and lead citrate and then visualized on a JEM 1400 electron microscope (JEOL) operated at $100 \mathrm{kV}$. Images were recorded with a Veleta $2 \mathrm{~K} \times 2 \mathrm{~K}$ digital camera (Olympus-SIS).

Statistics. Statistical analysis was performed with GraphPad Prism 7.0 software. All data are reported as mean \pm SEM. Statistical analysis was performed with 1-way ANOVA followed by Dunnett's post-hoc test when comparing more than 2 groups of data (Figure $1 \mathrm{H}$ and Figure $2 \mathrm{G}$ ) and by unpaired $t$ test when comparing 2 groups of data (Supplemental Figure 1). Data were considered statistically significant when a value of at least $P<0.05$ was achieved.

Study approval. All of the studies were performed according to protocols approved by the Weill Cornell Institutional Animal Care and Use Committee. 


\section{Author contributions}

ADL, AM, and YZ designed the studies. AM, YZ, LR, MAR, and JEI conducted experiments and acquired data. ADL, AM, YZ, MAR, and JEI analyzed data. ADL and AM wrote the manuscript. JEI contributed to the editing of the manuscript.

\section{Acknowledgments}

This work was supported by National Institute of Health grant R01HL126913 and the Harold S. Geneen Charitable Trust Awards Program for Coronary Heart Disease research to ADL. We are very thankful to Domenick J. Falcone and Roberto Levi for critical input and scientific discussion of the manuscript.

Address correspondence to: Annarita Di Lorenzo, Department of Pathology and Laboratory Medicine, Cardiovascular Research Institute, Brain and Mind Research Institute, Weill Cornell Medicine, 1300 York Avenue, New York, New York, 10065, USA. Phone: 212.746.6476; Email: and2039@med.cornell.edu.

1. Benjamin EJ, et al. Heart Disease and Stroke Statistics-2018 Update: A Report From the American Heart Association. Circulation. 2018;137(12):e67-e492.

2. Writing Group Members, et al. Heart Disease and Stroke Statistics-2016 Update: A Report From the American Heart Association. Circulation. 2016;133 (4):e38-360.

3. Nakashima Y, Plump AS, Raines EW, Breslow JL, Ross R. ApoE-deficient mice develop lesions of all phases of atherosclerosis throughout the arterial tree. Arterioscler Thromb. 1994;14 (1):133-140.

4. Getz GS, Reardon CA. Animal models of atherosclerosis. Arterioscler Thromb Vasc Biol. 2012;32(5):1104-1115.

5. Zadelaar S, et al. Mouse models for atherosclerosis and pharmaceutical modifiers. Arterioscler Thromb Vasc Biol. 2007;27(8):1706-1721.

6. Pendse AA, Arbones-Mainar JM, Johnson LA, Altenburg MK, Maeda N. Apolipoprotein E knock-out and knock-in mice: atherosclerosis, metabolic syndrome, and beyond. J Lipid Res. 2009;50 Suppl:S178-S182.

7. Abbott RD, Wilson PW, Kannel WB, Castelli WP. High density lipoprotein cholesterol, total cholesterol screening, and myocardial infarction. The Framingham Study. Arteriosclerosis. 1988;8 (3):207-211.

8. Ishibashi S, Brown MS, Goldstein JL, Gerard RD, Hammer RE, Herz J. Hypercholesterolemia in low density lipoprotein receptor knockout mice and its reversal by adenovirus-mediated gene delivery. J Clin Invest. 1993;92 (2):883-893.

9. Bentzon JF, Falk E. Atherosclerotic lesions in mouse and man: is it the same disease? Curr Opin Lipidol. 2010;21 (5):434-440

10. Braun A, et al. Loss of SR-BI expression leads to the early onset of occlusive atherosclerotic coronary artery disease, spontaneous myocardial infarctions, severe cardiac dysfunction, and premature death in apolipoprotein E-deficient mice. Circ Res. 2002;90 (3):270-276.

11. Caligiuri G, Levy B, Pernow J, Thorén P, Hansson GK. Myocardial infarction mediated by endothelin receptor signaling in hypercholesterolemic mice. Proc Natl Acad Sci USA. 1999;96 (12):6920-6924.

12. Van der Donckt $C$, et al. Elastin fragmentation in atherosclerotic mice leads to intraplaque neovascularization, plaque rupture, myocardial infarction, stroke, and sudden death. Eur Heart J. 2015;36 (17):1049-1058.

13. Kuhlencordt PJ, et al. Accelerated atherosclerosis, aortic aneurysm formation, and ischemic heart disease in apolipoprotein E/ endothelial nitric oxide synthase double-knockout mice. Circulation. 2001;104 (4):448-454.

14. Fernández-Hernando C, et al. Loss of Akt1 leads to severe atherosclerosis and occlusive coronary artery disease. Cell Metab. 2007;6 (6):446-457.

15. Stone NJ, et al. 2013 ACC/AHA guideline on the treatment of blood cholesterol to reduce atherosclerotic cardiovascular risk in adults: a report of the American College of Cardiology/American Heart Association Task Force on Practice Guidelines. Circulation. 2014;129(25 Suppl 2):S1-45.

16. Berry JD, Lloyd-Jones DM, Garside DB, Greenland P. Framingham risk score and prediction of coronary heart disease death in young men. Am Heart J. 2007;154 (1):80-86.

17. Liu D, et al. Smooth Muscle Hypoxia-Inducible Factor $1 \alpha$ Links Intravascular Pressure and Atherosclerosis--Brief Report. Arterioscler Thromb Vasc Biol. 2016;36(3):442-445.

18. Doran AC, Meller N, McNamara CA. Role of smooth muscle cells in the initiation and early progression of atherosclerosis Arterioscler Thromb Vasc Biol. 2008;28 (5):812-819.

19. Cheruvu PK, et al. Frequency and distribution of thin-cap fibroatheroma and ruptured plaques in human coronary arteries: a pathologic study. J Am Coll Cardiol. 2007;50 (10):940-949.

20. Braunwald E. Coronary plaque erosion: recognition and management. JACC Cardiovasc Imaging. 2013;6 (3):288-289.

21. Franck G, et al. Flow Perturbation Mediates Neutrophil Recruitment and Potentiates Endothelial Injury via TLR2 in Mice: Implications for Superficial Erosion. Circ Res. 2017;121 (1):31-42.

22. Crea F, Libby P. Acute Coronary Syndromes: The Way Forward From Mechanisms to Precision Treatment. Circulation. 2017;136 (12):1155-1166.

23. Shibata T, et al. Prevalence, Clinical Features, and Prognosis of Acute Myocardial Infarction Attributable to Coronary Artery Embolism. Circulation. 2015;132(4):241-250.

24. Prizel KR, Hutchins GM, Bulkley BH. Coronary artery embolism and myocardial infarction. Ann Intern Med. 1978;88(2):155-161.

25. Daugherty A, et al. Recommendation on Design, Execution, and Reporting of Animal Atherosclerosis Studies: A Scientific Statement From the American Heart Association. Arterioscler Thromb Vasc Biol. 2017;37(9):e131-e157.

26. Masucci-Magoulas L, et al. A mouse model with features of familial combined hyperlipidemia. Science. 1997;275 (5298):391-394

27. Pennacchio LA, et al. An apolipoprotein influencing triglycerides in humans and mice revealed by comparative sequencing. Science. 
2001;294 (5540):169-173.

28. Maxwell KN, Breslow JL. Proprotein convertase subtilisin kexin 9: the third locus implicated in autosomal dominant hypercholesterolemia. Curr Opin Lipidol. 2005;16 (2):167-172.

29. Lagace TA, et al. Secreted PCSK9 decreases the number of LDL receptors in hepatocytes and in livers of parabiotic mice. $J$ Clin Invest. 2006;116 (11):2995-3005.

30. Majesky MW. Developmental basis of vascular smooth muscle diversity. Arterioscler Thromb Vasc Biol. $2007 ; 27$ (6):1248-1258.

31. Bennett MR, Sinha S, Owens GK. Vascular Smooth Muscle Cells in Atherosclerosis. Circ Res. 2016;118(4):692-702.

32. Emini Veseli B, et al. Animal models of atherosclerosis. Eur J Pharmacol. 2017;816:3-13.

33. von Scheidt M, et al. Applications and Limitations of Mouse Models for Understanding Human Atherosclerosis. Cell Metab. 2017;25 (2):248-261.

34. Daugherty A. Mouse models of atherosclerosis. Am J Med Sci. 2002;323(1):3-10.

35. Matoba T, Sato K, Egashira K. Mouse models of plaque rupture. Curr Opin Lipidol. 2013;24 (5):419-425.

36. Seidell JC, Verschuren WM, Kromhout D. Prevalence and trends of obesity in The Netherlands 1987-1991. Int J Obes Relat Metab Disord. 1995;19 (12):924-927.

37. Franklin SS, Wong ND. Hypertension and cardiovascular disease: contributions of the Framingham heart study. Glob Heart. 2013;8(1):49-57.

38. Kannel WB, Castelli WP, Gordon T. Cholesterol in the prediction of atherosclerotic disease. New perspectives based on the Framingham study. Ann Intern Med. 1979;90 (1):85-91.

39. Newman WP, et al. Relation of serum lipoprotein levels and systolic blood pressure to early atherosclerosis. The Bogalusa Heart Study. NEngl J Med. 1986;314 (3):138-144.

40. Dahabreh IJ, Paulus JK. Association of episodic physical and sexual activity with triggering of acute cardiac events: systematic review and meta-analysis. JAMA. 2011;305 (12):1225-1233.

41. Smyth A, et al. Physical Activity and Anger or Emotional Upset as Triggers of Acute Myocardial Infarction: The INTERHEART Study. Circulation. 2016;134(15):1059-1067.

42. Stary HC, et al. A definition of initial, fatty streak, and intermediate lesions of atherosclerosis. A report from the Committee on Vascular Lesions of the Council on Arteriosclerosis, American Heart Association. Circulation. 1994;89(5):2462-2478.

43. Stary HC, et al. A definition of the intima of human arteries and of its atherosclerosis-prone regions. A report from the Committee on Vascular Lesions of the Council on Arteriosclerosis, American Heart Association. Circulation. 1992;85(1):391-405.

44. Ruan XZ, et al. Mechanisms of dysregulation of low-density lipoprotein receptor expression in vascular smooth muscle cells by inflammatory cytokines. Arterioscler Thromb Vasc Biol. 2006;26 (5):1150-1155.

45. Matsumoto K, et al. Expression of macrophage (Mphi) scavenger receptor, CD36, in cultured human aortic smooth muscle cells in association with expression of peroxisome proliferator activated receptor-gamma, which regulates gain of Mphi-like phenotype in vitro, and its implication in atherogenesis. Arterioscler Thromb Vasc Biol. 2000;20 (4):1027-1032.

46. Takahashi M, et al. Interleukin-1beta attenuates beta-very low-density lipoprotein uptake and its receptor expression in vascular smooth muscle cells. J Mol Cell Cardiol. 2005;38 (4):637-646.

47. Johnson J, et al. Plaque rupture after short periods of fat feeding in the apolipoprotein E-knockout mouse: model characterization and effects of pravastatin treatment. Circulation. 2005;111 (11):1422-1430.

48. Johnson JL, Jackson CL. Atherosclerotic plaque rupture in the apolipoprotein E knockout mouse. Atherosclerosis. 2001;154(2):399-406.

49. Calara F, Silvestre M, Casanada F, Yuan N, Napoli C, Palinski W. Spontaneous plaque rupture and secondary thrombosis in apolipoprotein E-deficient and LDL receptor-deficient mice. J Pathol. 2001;195 (2):257-263.

50. Chen YC, et al. A novel mouse model of atherosclerotic plaque instability for drug testing and mechanistic/therapeutic discoveries using gene and microRNA expression profiling. Circ Res. 2013;113 (3):252-265.

51. Ylä-Herttuala S, et al. Stabilisation of atherosclerotic plaques. Position paper of the European Society of Cardiology (ESC) Working Group on atherosclerosis and vascular biology. Thromb Haemost. 2011;106 (1):1-19.

52. Virmani R, Kolodgie FD, Burke AP, Farb A, Schwartz SM. Lessons from sudden coronary death: a comprehensive morphological classification scheme for atherosclerotic lesions. Arterioscler Thromb Vasc Biol. 2000;20 (5):1262-1275.

53. Lee RT, Libby P. The unstable atheroma. Arterioscler Thromb Vasc Biol. 1997;17(10):1859-1867.

54. Gough PJ, Gomez IG, Wille PT, Raines EW. Macrophage expression of active MMP-9 induces acute plaque disruption in apoE-deficient mice. J Clin Invest. 2006;116 (1):59-69.

55. Shankman LS, et al. KLF4-dependent phenotypic modulation of smooth muscle cells has a key role in atherosclerotic plaque pathogenesis. Nat Med. 2015;21(6):628-637.

56. Sambuceti G, et al. Coronary vasoconstriction during myocardial ischemia induced by rises in metabolic demand in patients with coronary artery disease. Circulation. 1997;95 (12):2652-2659.

57. Pupita G, et al. Myocardial ischemia caused by distal coronary-artery constriction in stable angina pectoris. N Engl J Med. 1990;323 (8):514-520.

58. Crossman DC. The pathophysiology of myocardial ischaemia. Heart. 2004;90(5):576-580.

59. Raphael CE, et al. Coronary Embolus: An Underappreciated Cause of Acute Coronary Syndromes. JACC Cardiovasc Interv. 2018;11 (2):172-180.

60. Véniant MM, Withycombe S, Young SG. Lipoprotein size and atherosclerosis susceptibility in Apoe (-/-) and Ldlr (-/-) mice. Arterioscler Thromb Vasc Biol. 2001;21 (10):1567-1570.

61. Bjørklund MM, et al. Induction of atherosclerosis in mice and hamsters without germline genetic engineering. Circ Res. 2014;114 (11):1684-1689.

62. Zhang Y, et al. Endothelial Nogo-B regulates sphingolipid biosynthesis to promote pathological cardiac hypertrophy during chronic pressure overload. JCI Insight. 2016;1 (5):e85484.

63. Lang RM, et al. Recommendations for chamber quantification: a report from the American Society of Echocardiography's Guidelines and Standards Committee and the Chamber Quantification Writing Group, developed in conjunction with the European Association of Echocardiography, a branch of the European Society of Cardiology. J Am Soc Echocardiogr. 
2005;18(12):1440-1463

64. Liu C, Wilson SJ, Kuei C, Lovenberg TW. Comparison of human, mouse, rat, and guinea pig histamine H4 receptors reveals substantial pharmacological species variation. J Pharmacol Exp Ther. 2001;299 (1):121-130. 